\title{
Erratum: Insight into the Microscopic Structure of an AdS Black Hole from a Thermodynamical Phase Transition [Phys. Rev. Lett. 115, 111302 (2015)]
}

Shao-Wen Wei and Yu-Xiao Liu

(Received 17 February 2016; published 22 April 2016)

DOI: 10.1103/PhysRevLett.116.169903

In the original Letter, there were two errors. The line element of the Ruppeiner geometry has a minus sign

$$
d s^{2}=-\frac{\partial^{2} S}{\partial x^{\mu} \partial x^{\nu}} \Delta x^{\mu} \Delta x^{\nu}
$$

and the thermodynamic scalar curvature should be

$$
R=-\frac{\left(n / n_{c}\right)^{6}-3\left(n / n_{c}\right)^{4}}{3 \pi\left[\left(n / n_{c}\right)^{4}-6\left(n / n_{c}\right)^{2}-3 P / P_{c}\right]}
$$

Then, Fig. 4 in the original Letter will be changed to Fig. 1.

After this correction, for the small black hole with $\left(n / n_{c}\right) \in\left(\sqrt{3}, \sqrt{3+\sqrt{3} \sqrt{3+P / P_{c}}}\right), R$ is positive and so the interaction between two molecules will be repulsive. Other results remain unchanged.

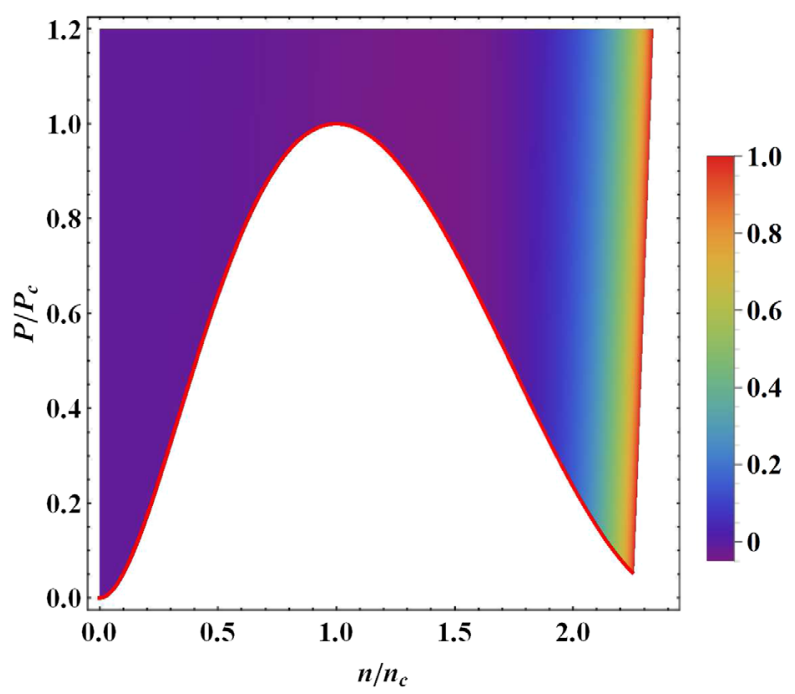

FIG. 1. The Ruppeiner scalar curvature $R$ as a function of the pressure $P$ and number density $n$. The boundary marked as the thick red line is related to the coexistence curve.

We are grateful to A. Dehyadegari, S. Hajkhalili, M. Kord Zangeneh, and A. Sheykhi for pointing out the errors. 